\title{
La cysticercose bovine en milieu sédentaire et en milieu nomade
}

\author{
par M. GRABER ef R. TABO
}

\begin{abstract}
RÉSUMÉ
Les auteurs éfudient, au Tchad, la Cysticercose bovine en milieu sédeniaire et en milieu nomade. lls constatent qu'cu Batha (Centre Tchad), il existe 4 fois plus d'animaux ladres chez les sédentaires que chez les nomades.

Au Kanem (Ouest Tchad), la proportion est à peu près identique. Dans les 2 préfectures le taux d'infestation du bétail nomade est très voisin (environ 5 p. 100).
\end{abstract}

\section{INTRODUCTION}

La Cysticercose bovine constitue au Tehad un problème économique d'une grande importance ef deux études lui ont été déjà consacrées (GRABER 1959 ; GRABER et THOME 1964).

Elles ont permis d'apporter un certain nombre de précisions sur les conditions qui favorisent le développement de cette maladie dans les zones sèches du Tchad qui sont également les régions où vivent les troupeaux les plus nombreux.

Cependant, une question n'a été que très partiellement abordée : celle de la répartition de la ladrerie bovine en milieu sédentaire et en milieu nomade.

Ce problème mérite de retenir plus particulièrement l'attention.

\section{CONDITIONS DE L'ENQUETTE}

Cette enquête a été menée au Kanem (Ouest Tchad) et 'au Batha (Centre Tchad). Elle a duré 11 mois, de juillet 1963 à mai 1964 et a porté sur 122 animaux de boucherie adultes dans le premier cas et 397 dans le second.

Les zébus du Batha ont été examinés aux abattoirs d'Ati et de Koundjourou qui était, à l'époque, un gros centre de fabrication de viande séchée. Ceux du Kanem l'ont été dans différents villages dépendant de la préfecture de Mao et de la sous-préfecture de Moussoro.
La viande des animaux a été découpée en menus morceaux et les Cysticerques ont été soigneusement recherchés dans les muscles et les organes, récoltés, mis en flacon et formolés en vue de leur examen ultérieur.

En même temps, les enquêteurs se sont renseignés sur l'origine des animaux et sur leurs déplacements habituels, ce qui, en pleine zone d'Elevage, présente un peu moins de difficulté que dans les grands centres d'abattage.

Dans un stade ultıme, au laboratoire, les Cystıcerques ont été mis en coupes sériées et leur âge apprécié.

\section{RÉSULTATS}

10 Résultats d'ensemble.

Ils figurent aux tableaux no 1 ef 2 . Pour le Batha, le taux d'infestation moyen' est un peu mans important que celui des années 1958-1960 au cours desquelles un nombre d'animaux sédentaıres beaucoup plus élevé avait été autopsié.

L'enquête confirme le fait que le bétail nomade est quatre fois moins parasité que le bétail sédentaire. Il est bon de préciser que dans le cas du Batha, Il s'agissait en général de «grands» transhumants qui n'hésitent pas à se déplacer de l'Ouaddi Haddad dans le Nord jusqu'au fond du Salamat et du Moyen Chari dans le Sud, soit sur une distance de plusieurs centaines de kilomètres. 
CYSTICERCOSE BOVINE EN MILIEU SEDENTAIRE ET EN MILIEU NOMAOE

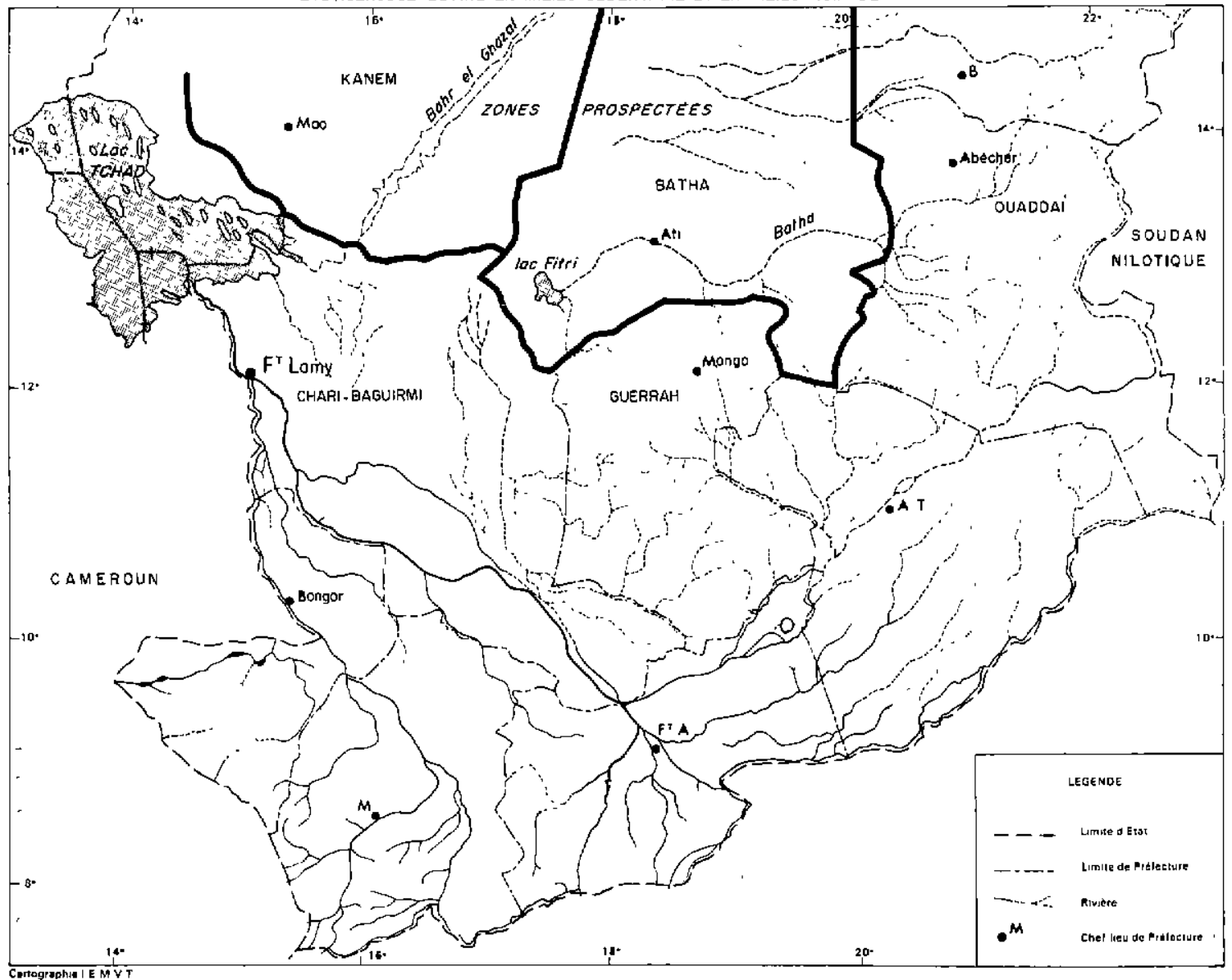

TABLEAU $\mathrm{N}^{\circ} \mathrm{I}$

Pourcentage d'infestation des zêbus du Batha

\begin{tabular}{|l|c|c|c|}
\hline & Nomades & Sêdentaires & Total \\
\hline Nombre d'animaux autopsiës & 339 & 58 & 397 \\
\hline Nombre d'animaux parasitës & 18 & 12 & 30 \\
\hline Taux d'infestation & 5,3 p.100 & $20 \mathrm{p} .100$ & $7,5 \mathrm{p} .100$ \\
\hline Arnées I958-1960 & 34 sur 317 autopsiés soit $10,7 \mathrm{p} .100$ \\
\hline
\end{tabular}

TABLEAU N $N^{\circ}$ II

Pourcentage d'infestation des zébus du Kanem

\begin{tabular}{|l|c|c|c|}
\hline & Nomades & Sédentaires & Total \\
\hline Nombre d'animaux autopsiĕs & 99 & 23 & 122 \\
\hline Nombre d'animaux parasités & 5 & 1 & 6 \\
\hline Taux d'infestation & 5,05 p.100 & 4,3 p.100 & 4,9 p.100 \\
\hline Annếes $1957-1962$ & 9 sur 183 & soit 4,9 p.100 & \\
\hline
\end{tabular}


En ce qui concerne le Kanem, les chiffres obtenus tant chez les sédentaires que chez les nomades sont à peu de choses près superposables et ne diffèrent guère de ceux recueillis au cours d'une enquête précédente (1957-1963). Au Kanem, les transhumances sont dans l'ensemble, beaucoup plus limitées qu'au Batha.

Chose curieuse, tant au Kanem qu'au Batha, on retrouve chez les animaux appartenant à des éleveurs nomades des pourcentages de Cysticercose très voisins $(5,05$ p. $100 ; 5,3$ p. 100).

II est difflcile d'expliquer cette distorsion entre transhumants et sédentaires peut-on invoquer du Centre Tchad. Tout au plus peut-on invoquer en milieu nomade un certain relachement de la symbiose homme-bétail qui conditionne l'apparition de la Cysticercose dans un troupeau : les hommes sont moins nombreux le bétail plus dispersé et plus mobile, moins concentré autour des zones d'habitation qu'en zone de peuplement sédentaire.

Quant au téniasis humain, on ne dispose que de renseignements globaux. Selon une statistique datant de 1964, le nombre d'individus atteints serait d'environ 5 p. 100 (Anonyme 1964).

Le Kanem est une régıon traditionnellement peu touchée par la Cysticercose. Le mode d'élevage en raison des faibles transhumances varie tout compte fait assez peu, ce qui entraîne une certaıne uniformité dans le taux d'infestation par Cysticercus bovis.

\section{$2^{0}$ Stade d'évolution des Cysticerques.}

Les tableaux 3 ef 4 résument les observations faites en ce qui concerne, la localisation des parasites, leur nombre ct leur stade d'évolution chez les zébus du Centre Tchad.

Il apparaît que, les Cystıcerques récoltés chez des animaux transhumants âgés de plus de 8 ans sont, pour les trois quarts d'entre eux tous calcifiés. Par contre chez les sédentaires, la proportion n'est plus que de la moitié.

Par ailleurs, le nombre de parasites par zébu semble plus important dans le second groupe que dans le premier.

Il en résulte que les animaux âgés qui, pour la plupart, sont abattus sur des marchés de brousse sans inspection, donc sans stérilisation, représentent la meilleure source d'infestation pour l'homme qui les consomime et assurent l'entretien continu du téniasis humain.

Au Kanem, les Cysticerques examinés étalent tous calcifiés, que ce soit chez les sédentaires ou chez les nomades.

\section{$3^{\circ}$ Epoque des infestations.}

Les résultats du tableau 5 montrent que pour la période considérée les mols de mars-avril et septembre-octobre sont ceux où l'infestation est maximale.

Un cutre sondage en 1965 à l'abattoir d'Ati confirme le fait.

Ces constatations ne coincident pas exactement avec ce qui avait été écrit précédemment (GRABER ef THOME 1964). II est vrai que la première enquête (1958-1960) intéressait des animaux un peu plus jeunes, plus parasités et provenant, dans 60 p. 100 des cas, d'élevages sédentaires. La Cysticercose paraissait, dans ces conditions, un peu moins fréquente en mars et en mai. Il est probable que la situation varie selon les années et la proportion de bétail sédentaire ou nomade qui entre dans les statistiques.

Cependant, aux abattoirs de Fort-Lamy, depuis déjà plusieurs années, au moment de la «soudure » c'est-à-dire à partir d'avril, les expéditeurs de viande cessent de s'approvisionner au Batha, tant pour des ralsons pratiques (transport) que pour des raisons sanitaires dont la principale est l'existence à cette époque de l'année d'une forte proportion d'animaux ladres.

Au Kanem, la Cysticercose est surtout une affection d'hivernage (à partir de juin) ce qui corrobore les observations précédentes (GRABER et THOME, 1964).

\section{CONCLUSIONS}

519 zébus de boucherle âgés ont, de juillet 1963 à juin 1964, été autopsiés au Batha (Centre: Tchad) et au Kanem (Ovest Tchad). Les Cysti-! cerques ont été systématiquement recherchés et leur âge apprécié. Les constatations suivantes. ont été faites :

10 II existe quatre fois plus d'animaux ladres chez les sédentaires du Batha que chez les nomades, alors qu'au Kanem la proportion est à peu près identique.

$2^{\circ}$ Le taux d'infestation du bétail nomade dans. 
Retour au menu

TABLEAU $\mathrm{N}^{\circ}$ III

Stade d'êvolution des cysticerques chez les zêbus nomades du Batha

\begin{tabular}{|c|c|c|c|c|}
\hline Age des zëbus & Nombre d'animaux & Nombre de cysticerques & Localisation & Etat des cysticerques \\
\hline 3 ans & 1 & 2 & langue & vivants \\
\hline 8 ans & 2 & 2 à 3 & langue & tous calcifiês \\
\hline 9 ans & 2 & 1 à 5 & $\begin{array}{l}\text { langue } \\
\text { ếpaule }\end{array}$ & $\begin{array}{l}1 \text { vivant } \\
\text { calcifiés }\end{array}$ \\
\hline 10 ans & 2 & 1 à 6 & langue & calctfiếs \\
\hline 12 ans & 1 & 1 & langue & calcifié \\
\hline Hors d’âge & 10 & 1 à 9 & $\begin{array}{l}\text { g langue } \\
1 \text { épaule }\end{array}$ & $\begin{array}{l}7 \text { calcifiés } \\
\text { vivants }\end{array}$ \\
\hline
\end{tabular}

TABLEAU $\mathrm{N}^{\circ}$ IV

Stade d'êvolution des cysticerques chez les zêbus sédentaires du Batha

\begin{tabular}{|c|c|c|c|c|}
\hline Age des zêbus & Nombre d'animaux & Nombre de cysticerques & Localisation & Etat des cysticerques \\
\hline 7 ans & 2 & 2 à 3 & $\begin{array}{l}\text { masseters } \\
\text { 1angue }\end{array}$ & $\begin{array}{l}1 \text { vivant } \\
2 \text { calcifiés }\end{array}$ \\
\hline 8 ans & I & 3 & langue & calcifiés \\
\hline 9 ans & 2 & 3 à 23 & $\begin{array}{l}\text { langue } \\
\text { épaule }\end{array}$ & vivants \\
\hline 10 ans & 1 & 3 & coeur & vivants \\
\hline 11 ans & 1 & 2 & langue & calcifiës \\
\hline Hors d'âge & 5 & 1 à 5 & $\begin{array}{l}\text { langue } \\
\text { epatile } \\
\text { psoas }\end{array}$ & $\begin{array}{l}3 \text { calcifiês } \\
2 \text { vivants }\end{array}$ \\
\hline
\end{tabular}

TABLEAU $\mathrm{N}^{\circ} \mathrm{V}$

Epoque des infestations (Batha)

\begin{tabular}{|c|c|c|c|c|}
\hline \multirow{2}{*}{ Epoquc } & \multicolumn{2}{|c|}{ Nombre d'animaux autopsiés } & \multicolumn{2}{|c|}{ Nombre d'animaux parasités } \\
\hline & nomades & sêdentaires & nomades & sédentaires \\
\hline Juillet 1963 & 4 & 6 & 0 & 0 \\
\hline août 1963 & 24 & 3 & 0 & 0 \\
\hline septembre 1963 & 40 & 12 & 1 & 2 \\
\hline octobre 1963 & 56 & 9 & 0 & 2 \\
\hline novembre 1963 & 39 & 3 & 1 & 0 \\
\hline décembre 1963 & 37 & 2 & 0 & 0 \\
\hline février 1964 & 10 & - & 0 & - \\
\hline mars 1964 & 58 & 13 & 5 & 4 \\
\hline avril 1964 & 59 & 10 & 10 & 4 \\
\hline $\operatorname{ma1} 1964$ & 12 & - & 1 & - \\
\hline Total & 339 & 58 & 18 & 12 \\
\hline
\end{tabular}


les deux régions considérées est très voisin (un peu plus de 5 p. 100).

$3^{\circ}$ Les trois quarts des animaux nomades sont, au Batha, porteurs de Cysticerques calcifiés, contre 50 p. 100 seulement chez les sédentarres. Au Kanem il n'a été vu au cours de cette enquête que des parasites dégénérés. La proportion est la même dans les deux cas.

40 Le plus grand nombre de Cysticerques a été rencontré au Kanem en début d'hivernage. Au Batha, lorsqu'il s'agit de lots d'animaux où le bétail nomade est en majorité, l'infestation semble maximale en mars-avril.

50 Les auteurs insistent sur le danger que présente pour la santé humaine la viande des anımaux âgés, viande souvent ladre et mise en consommation sur place, dans les marchés de brousse, sans inspection et sans stérilisation.

\section{SUMMARY}

The bovine cysticercosis amongst sedenlary and nomadic cattle

Bovine cysticercosis has been studied amongst sedentary and nomadic cattle, in Chad. In Batha (Centre Chad) the sedentary cattle have been reported to be 4 times more infestated by $C y s t i c e r c o s i s$ than the nomodic anumals.

In Kanem (West-Chad), the infestation rate of both nomadic and sedentary cattle is nearly the same. In bath prefectures, the infestation rate of nomadic cattle is very similar (about 5 p. 100).

\section{RESUMEN}

La Cisticercosis de los bovinos en medio sedentario y nomade

Los autores estudian, en Chad, la cisticercosis de los bovinos en medio sedentario y nomade. Según sus observaciones, se encuentran en Batha (Centro de Chad) 4 veces más de animales triquinosos en los sedentarios que en los nomades.

En Kanem (Oeste de Chad), el termino medio es casi igual. En los dos prefecturas, el porcentaje de infestación del ganado nomade es muy cercano (como 5 p. 100).

\section{BIBLIOGRAPHIE}

GRABER (M.). - La cysticercose bovine. Son importance dans les zones sahéliennes d'Elevage de la République du Tchad. Rev. Elev. Méd. Vét. pays trop., 1959, 12, 2, 121-145.

GRABER (M.) et THOME (M.). - La cysticercose en République du Tchad. Quelques réflexions sur la situation présente, l'étiologie, le diagnostic, l'immunité et le traitement de cette zoonose. Rev. Elev. Méd. Vét. pays trop., 1964, 17, 3, 441-466.

ANONYME. - Rapport annuel laboratoire de Farcha, 1964, 151-153.

ANONYME, - Rapport annuel laboratoire de Farcha, 1965, 125-6. 